\title{
The Effect of an Isometric Hip Muscle Strength Training Protocol on Valgus Angle During a Drop Vertical Jump in Competitive Female Volleyball Players
}

\author{
Kaitlin M. Jackson ${ }^{1}$, Tyson A. C. Beach ${ }^{2 *}$, David M. Andrews ${ }^{1}$ \\ ${ }^{1}$ Department of Kinesiology, University of Windsor, 401 Sunset Avenue, Windsor, ON, Canada N9B 3P4, ${ }^{2}$ Faculty of Kinesiology \& \\ Physical Education, University of Toronto, 55 Harbord St, Toronto, ON, Canada M5S 2W6
}

Corresponding Author: Tyson A. C. Beach, E-mail: tyson.beach@utoronto.ca

\section{ARTICLE INFO}

Article history

Received: August 13, 2017

Accepted: September 29, 2017

Published: October 31, 2017

Volume: 5 Issue: 4

Conflicts of interest: None

Funding: None

\begin{abstract}
Background: Hip muscle weakness is associated with higher peak knee valgus angles (VA) during drop vertical jumps (DVJ) and linked to ACL injury risk. Objective: To determine if isometric strengthening (IST) of the hip extensor, abductor, and external rotator muscle groups would reduce VA exhibited during a DVJ. Methodology: Fourteen female volleyball players (7 training (TG), 7 control (CG), VA $\geq 9^{\circ}$ during DVJ) participated. Pre- and post-test gluteal, quadriceps and hamstring strength were measured with a digital force gauge. Three-dimensional kinematics were collected during 15 DVJ trials. TG participated in a 6-week IST program that targeted the hip extensor, abductor, and external rotator muscle groups. Two-way mixed ANOVAs compared mean differences of VA and strength. Single-participant analyses examined if athlete-specific adaptations went undetected in the analyses of aggregated data. Results: TG hip extension, abduction, and knee flexion strength increased, respectively, by $20.5 \%, 27.5 \%$ and $23.5 \%(\mathrm{P}<0.05)$. No group-level changes in VA were detected. Unilateral VA decreased for 5 TG participants, and bilateral VA decreased in 2 TG participants. Conclusions: IST increased isometric hip muscle strength, but its effect on VA is inconclusive based on group-level analyses. Using single-participant designs, future studies should assess IST and/or dynamic resistance/ neuromuscular training in a larger sample to determine its effect on ACL injury risk factors.
\end{abstract}

Key words: Anterior Cruciate Ligament, Knee Injuries, Sports, Training, Kinematics

\section{INTRODUCTION}

Preventing anterior cruciate ligament (ACL) injuries in female athletes has been heavily researched in recent decades due to high injury rates. Female athletes have twice as many ACL injuries (de Loes, Dahlstedt, \& Thomee, 2000) and require surgery for ligamentous knee injuries almost twice as often as their male counterparts (Fernandez, Yard, \& Comstock, 2007). Female athlete knee injuries predominantly occur during non-contact maneuvers such as deceleration, pivoting, or landing tasks that are associated with high external loads at the knee joint (Bessier, Lloyd, Cochrane, \& Ackland, 2001; Boden, Dean, Feagin, \& Garrett, 2000; Hewett et al., 2005). There has been an emphasis in the literature placed on sports-related non-contact injuries sustained during landing activities due to the high forces and high risk biomechanical strategies used (Chappell \& Limpisvasti, 2008; Ford, Myer, \& Hewett, 2003; Hewett et al., 2005; Jacobs, Uhl, Mattacola, Shapiro, \& Rayens, 2007; Joseph et al., 2008; Myer, Ford, McLean, \& Hewett, 2006). These high-risk landing strategies have prompted a focus on exercise interventions in an attempt to alter landing mechanics and subsequently reduce injury risk (Cammarata \& Dhaher, 2010; Ford et al., 2003;
Howard, 2011; Jacobs et al., 2007; Joseph et al., 2011; Myer, Ford, Palumbo, \& Hewett, 2005).

The ACL's primary role in knee stabilization is to resist both anterior translation and internal rotation of the tibia relative to the femur (Butler, Noyes, \& Grood, 1980; Ellison \& Berg, 1985). It is the positive association of knee valgus motion with the occurrence of anterior tibial translation and internal rotation that relates valgus motion to increased knee ligament strain and injury potential (Cooke, Sled, \& Scudamore, 2007; Myer et al., 2005). In a non-contact landing, high knee valgus deviation (frontal plane projection angle of $9^{\circ}$ or greater) has been found to be one of the strongest links to ACL injury (Hewett et al., 2005; Levine et al., 2013).

Recent research in this area has focused on the effectiveness of various exercise interventions to improve the biomechanics of the knee by strengthening hip muscles (Anwer \& Alghadir, 2014; Earl-Boehm \& Hoch, 2011; Herman et al., 2008). With the hip being the most proximal joint of the lower extremity closed-kinetic-chain, attaining adequate control of this joint is hypothesized to improve control at the knee (Powers, 2010). Evidence of this is seen with a reported link between hip muscle strength and non-contact ACL injury 
(Khayambashi, Ghoddosi, Straub, \& Powers, 2015). Specifically, muscle weakness in hip abduction (Jacobs et al., 2007; Nadler et al., 2002), external rotation (Howard, 2011; Khayambashi, Mohammadkhani, Ghaznavi, Lyle, \& Powers, 2012), and extension (Nadler et al., 2002) are all associated with higher knee valgus angles during landing. Therefore, strengthening hip muscles to improve control of the femur proximally could help to maintain a more neutral alignment of the distal femur with the tibia at the knee, thereby preventing excessive valgus deviation.

Some studies on the effectiveness of dynamic resistance and/or neuromuscular (plyometrics, agility, balance) exercise protocols have shown that such interventions can improve drop vertical jump (DVJ) mechanics in controlled settings (Hewett et al., 2005; Myer et al., 2005), while other studies show strength improvements but no changes in lower extremity movement mechanics (Chappell et al., 2008; Ferber, Kendall, \& Farr, 2011). The inconsistencies in the results between studies could be due, in part, to the variation of exercises and exercise durations utilized.

Isometric resistance training of hip musculature has not been reported in the literature to improve DVJ mechanics. This type of training typically involves constant-length muscle contractions of targeted muscles surrounding one joint at a time, with the focus being on strengthening the muscles primarily responsible for resisting joint rotation when an external force is applied. Studies show that isometric resistance training produces larger strength gains (Duchateau \& Hainaut, 1984; Folland, Hawker, Leach, Little, \& Jones, 2005) and faster muscle activation timing (Tsao, Galea, \& Hodges, 2010) than dynamic resistance training. However, these results were joint position-specific and have yet to be shown to transfer to changes in DVJ mechanics. The majority of resistance training protocols investigated in the literature to date have used dynamic exercises to modify lower extremity biomechanics, in an attempt to improve injury outcomes. Although there is increasing evidence that some of these approaches are efficacious (Chappell et al., 2008; Ferber et al. 2011; Myer et al., 2005), the components of the interventions are inconsistent, leaving questions regarding their independent contributions and/or relative (in) effectiveness. A recent meta-analysis (Donnell-Fink et al., 2015) dubbed the general quality of these studies to be somewhat low, leaving room for future studies to assess the efficacy of the specific components of various interventions. The effect that isometric hip muscle strengthening alone has on ACL injury risk factors has yet to be studied.

Considering all the research completed to date, no study has assessed the effectiveness of an isometric tri-planar hip-strengthening program to control frontal plane knee motion during a DVJ task. Therefore, the purpose of this study was to determine if an isometric strengthening program targeting the hip extensor, abductor, and external rotator muscles would improve the VA during a DVJ. It was hypothesized that there would be an increase in hip joint moment-generating capacity (i.e. hip muscle strength) following isometric hip muscle training, which would result in a decrease in knee valgus motion during a DVJ.

\section{METHODOLOGY}

\section{Participants and Study Design}

Fourteen female volleyball players (age $22.4 \pm 1.6$ years, height $1.77 \pm 0.09 \mathrm{~m}$, and body mass of $69.5 \pm 10.2 \mathrm{~kg})(7$ training group [TG], 7 control group [CG]), with at least two years of experience on university, college and/or club level volleyball teams, were recruited from the Greater Toronto Area (Ontario, Canada). All participants had no history of lower extremity surgery, no lower extremity injury within 3 months of starting the study, and demonstrated a peak knee valgus angle of $9^{\circ}$ or greater (2D projected angle) during drop landings, as determined during a pre-screening protocol (detailed below). This study was approved by the Research Ethics Boards of both the University of Toronto and University of Windsor. Written informed consent was obtained from each participant prior to data collection.

The procedures of this study were executed in four stages described below: pre-screen; pre-test; training; and post-test. During the pre- and post-test data collection sessions, all participants performed 15 trials of a DVJ task approximately 7 weeks apart. The TG participated in 6 weeks $(5 \mathrm{x} /$ week) of isometric strength training between the pre- and post-test data collection sessions, while the CG continued normal activity during this period.

\section{Test Procedures}

The peak knee valgus angles of a total of 109 volleyball players were quantified from frontal plane video records obtained during a pre-screen session in order to determine eligibility for the full study. Players were eligible to participate in the study if the peak knee valgus angle (2D frontal projection angle) during at least two of three DVJ trials was $9^{\circ}$ or greater. During the screening session, participants wore spandex shorts, a t-shirt, and the shoes that they typically wear during volleyball participation. An Apple iPad tablet ( ${ }^{\text {rd }}$ Gen., Taiwan) was set up on a tripod at knee height, approximately two meters in front of each participant while they performed a DVJ (described below). The frame that corresponded with the highest knee valgus angle observed during the landing of the DVJ task (peak medial deviation) was used to determine eligibility in the study. Each potential participant performed a minimum of three practice trials to familiarize herself with the task. Three trials were recorded for each player with a minimum of 30 seconds rest provided between trials.

For the pre- and post-test data collection sessions, participants were asked to wear athletic shoes in which they would normally play volleyball, and a sports bra and spandex shorts, in order to minimize clothing interference during motion capture. Upon arrival to the laboratory, the participant's body mass $(\mathrm{kg})$ and height $(\mathrm{m})$ were measured when standing on a force platform with a reflective marker (for motion capture) placed at the apex of their head.

To measure the strength of the gluteal, hamstring and quadriceps muscle groups bilaterally, each participant performed three maximum voluntary isometric contraction (MVIC) trials against a digital force gauge (Manual Muscle Testing System, Layfayette Instrument Company, Lafayette, IN) anchored to a 
custom-made table (MotionBlock ${ }^{\mathrm{TM}}$, Toronto, Canada) which had built-in adjustable lever arms to provide the resistance against which the participants contracted (Figure 1).

Each MVIC trial involved a brief ramp up to a maximal effort contraction (held for 5 seconds), followed by a return to rest. A minimum of 60 seconds rest was provided between trials. The researcher provided verbal motivation to encourage maximal effort. Standardized positions of each force measurement for each muscle are outlined in Table 1.

Following the MVIC trials, a total of 45 spherical (14 mm) reflective markers were placed with double-sided tape on: top of the head; C7; sternal notch; xiphoid process; four skin markers placed asymmetrically approximately $5-10 \mathrm{~cm}$ from the spine at the $\mathrm{T} 5$ and $\mathrm{T} 9$ spinal processes; acromion processes; iliac crests; three on the lateral posterior pelvis bilaterally; greater trochanters; frontal thighs at the approximate midpoint of the thigh; lateral thighs halfway between the frontal thigh marker and the knee; medial and lateral femoral epicondyles; tibial tuberosities; anterior midpoints of the shanks; lateral shanks halfway between the anterior shank and ankle joint; medial malleolus; lateral malleolus; posterior calcaneus; intermediate cuneiforms; and the $1^{\text {st }}$ and $5^{\text {th }}$ metatarsals (Figure 2 ).

After the collection of a static calibration trial during

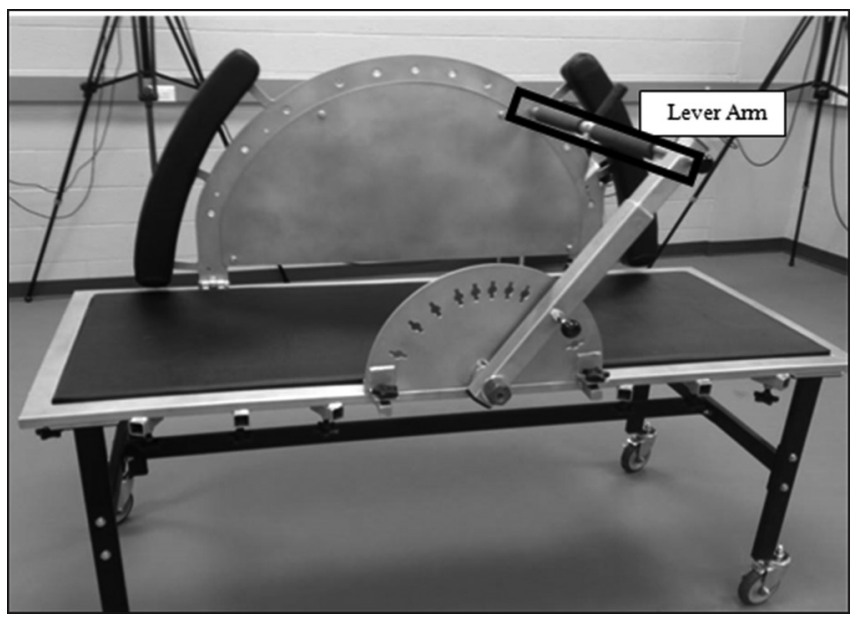

Figure 1. MotionBlock ${ }^{\mathrm{TM}}$ table used for MVIC and isometric strength training

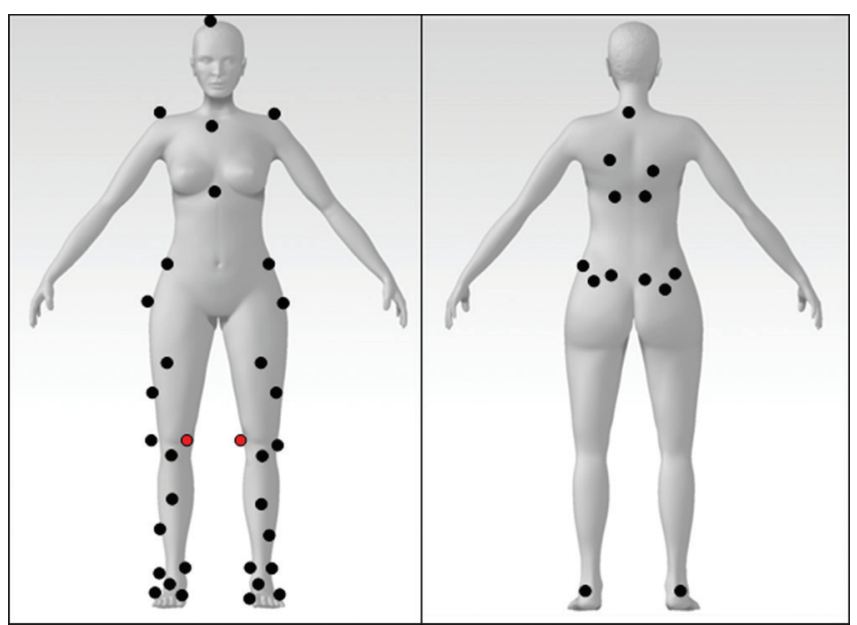

Figure 2. Reflective marker placement for 3D tracking (black dots) and calibration only (red dots) which participants stood "quietly" in the anatomical position, the medial knee markers were removed to prevent interference during the DVJ trials. Each DVJ trial began with participants standing atop a box $30 \mathrm{~cm}$ high, with their feet approximately shoulder-width apart. Participants were instructed to drop down (the stepping foot was not specified) with each foot landing on one of the two force platforms placed directly in front of the box. Immediately following the landing, participants executed a vertical jump with their shoulders fully extended, as if they were performing a maximal block jump. Given that DVJ mechanics are influenced by verbal cueing (Khuu, Musalem, \& Beach, 2015), participants were specifically instructed prior to stepping down to "drop down on the force platforms, then immediately jump up as if you are performing a maximal block jump. Focus on pressing your hands over a net, and land with one foot on each platform". The focus for the participant was directed to upper body block jump technique in order to simulate a practice/game situation, as well as to direct their attention away from their lower body mechanics. Several practice trials were permitted to ensure participants were comfortable with the DVJ task. A total of 15 trials were recorded for each participant, with a minimum of 60 seconds rest provided between trials.

An optoelectronic motion capture system (Qualisys AB, Gothenburg, Sweden) with eight Oqus 1 (0.3 MP) cameras collected 3D marker position data at $200 \mathrm{~Hz}$ during the DJV trials. $A \pm 10 \mathrm{~V}, 16$-bit analog-to-digital conversion system (Analog Acquisition Interface Unit, Qualisys AB, Gothenburg, Sweden) was used to synchronously collect force platform data at $1000 \mathrm{~Hz}$. Qualisys Track Manager (QTM) software was used to acquire marker position and force platform data. An MTD-2 CalTester Rod (Motion Lab Systems, Inc., Baton Rouge, LA) with CalTesterPlus software (C-Motion, Inc., Germantown, MD) was used to spatially locate two force platforms (AMTI-OR6-6-1000, AMTI, Waterdown, MA). Visual3D (C-Motion, Inc., Germantown, MD) was used to smooth marker position and force platform data using a zero-lag Butterworth low-pass filter with an effective cut-off frequency of $10 \mathrm{~Hz}$ (Kristianslund, Krosshaug, \& van den Bogert, 2012), and to generate a 3D dynamic linked-segment model of the body (as described in Khuu et al., 2015). From the linked-segment model, bilateral peak knee valgus angles were extracted for statistical analyses.

\section{Exercise Protocol}

The TG performed a 6-week isometric strength training protocol, consisting of five sessions per week (three supervised and two unsupervised), each lasting approximately 45 60 minutes. During the unsupervised sessions, the TG group was encouraged to train in groups to maximize compliance. There were two missed supervised sessions for four of the participants that occurred in different weeks, resulting in each of those participants having only two supervised and three unsupervised sessions for two of their training weeks.

Each exercise session included five isometric exercises (Table 2), which were completed as three sets of ten maximal effort isometric contractions lasting ten seconds (10 
Table 1. Standardized positions for the maximum isometric strength tests during pre- and post-training data collection sessions. The force gauge was attached to a lever at a fixed position tailored to each of the four positions

\begin{tabular}{ll}
$\begin{array}{l}\text { Target muscle/ } \\
\text { Muscle group }\end{array}$ & Position \\
\hline Gluteus Maximus & $\begin{array}{l}\text { The participant lay prone on the Motionblock } \\
\text { TMe table with knee flexed to } 90^{\circ} \text { and the ankle in a neutral position. } \\
\text { instructed to extend her thigh off the table while keeping her ASIS in contact with the table. The force gauge was } \\
\text { positioned at the midpoint between the popliteal fossa and the gluteal fold and resisted extension }\end{array}$ \\
& $\begin{array}{l}\text { The participant lay on the Motionblock } \\
\text { HM table on the contralateral side of the gluteus medius being measured. } \\
\text { Her hip was in a neutral position. The researcher applied pressure and visually monitored the participant's lower } \\
\text { back to prevent excessive motion. The participant was instructed to maximally abduct the leg as the force gauge } \\
\text { provided resistance. The force gauge was positioned } 4 \text { cm above the knee joint on the lateral aspect of the distal } \\
\text { thigh } \\
\text { The participant lay prone on the Motionblock }\end{array}$ \\
& $\begin{array}{l}\text { pressure to the participant's lower back to prevent hip extension and other excessive motion. The participant was } \\
\text { instructed to maximally flex the knee as the force gauge provided resistance. The force gauge was adjusted to } \\
\text { contact the skin at the posterior aspect of the distal shank at a knee flexion angle of } 90^{\circ}\end{array}$ \\
& $\begin{array}{l}\text { The participant was seated at the side of the MotionBlock } \\
\text { instructed to maximally extend the knee as the force gauge provided resistance. The force gauge was adjusted to } \\
\text { contact the skin at the frontal aspect of the distal shank at a knee flexion angle of } 90^{\circ}\end{array}$ \\
Quadriceps &
\end{tabular}

Table 2. Position, action and target muscles associated with the exercises completed during each training session. All positions were completed while lying supine, unless otherwise stated

\begin{tabular}{|c|c|c|c|}
\hline Exercise & Position & Action & Target muscles \\
\hline 1 & $\begin{array}{l}\text { Prone } \\
90^{\circ} \text { knee flexion } \\
\text { Ankle in neutral } \\
\text { position } \\
\text { No lateral } \\
\text { movement of } \\
\text { limb }\end{array}$ & Hip extension & $\begin{array}{l}\text { Gluteus maximus } \\
\text { Biceps femoris }\end{array}$ \\
\hline 2 & $\begin{array}{l}\text { Prone } \\
\text { Full hip external } \\
\text { rotation } \\
90^{\circ} \text { knee flexion } \\
30^{\circ} \text { hip } \\
\text { abduction } \\
\text { Ankle in neutral } \\
\text { position }\end{array}$ & Hip extension & $\begin{array}{l}\text { Gluteus maximus } \\
\text { Gluteus medius } \\
\text { Biceps femoris }\end{array}$ \\
\hline 3 & $\begin{array}{l}0^{\circ} \text { hip abduction } \\
0^{\circ} \text { hip flexion } \\
0^{\circ} \text { hip rotation }\end{array}$ & Hip abduction & $\begin{array}{l}\text { Gluteus maximus } \\
\text { Gluteus medius }\end{array}$ \\
\hline 4 & $\begin{array}{l}30^{\circ} \text { hip } \\
\text { abduction } \\
0^{\circ} \text { hip flexion } \\
\text { Full hip external } \\
\text { rotation }\end{array}$ & $\begin{array}{l}\text { Hip abduction } \\
\text { and external } \\
\text { rotation }\end{array}$ & $\begin{array}{l}\text { Gluteus maximus } \\
\text { Gluteus medius }\end{array}$ \\
\hline 5 & $\begin{array}{l}0^{\circ} \text { hip flexion } \\
90^{\circ} \text { knee flexion }\end{array}$ & Knee flexion & Biceps femoris \\
\hline
\end{tabular}

$\mathrm{s}$ rest between reps, $60 \mathrm{~s}$ rest between sets). Each exercise was performed bilaterally, one side at a time. Stationary resistance was met via the MotionBlock ${ }^{\mathrm{TM}}$ while maintaining prescribed position. Participants were coached on where they should expect to feel the contraction, based on the muscle group targeted in each exercise. If the participant felt a non-targeted muscle group becoming active during the contraction, they were instructed to stop and rest for one or two minutes and re-attempt the exercise. All participants verbally indicated that they were able to perform the exercises and feel the target muscle(s) activating.

For exercises 1 and 2, while lying in a prone position on the MotionBlock ${ }^{\mathrm{TM}}$ table, participants attempted to raise the thigh being measured off the MotionBlock ${ }^{\mathrm{TM}}$ table while keeping both anterior superior iliac spines firmly against the mat. If additional biofeedback was necessary to help the par- 
ticipant, light pressure was applied by the researcher's hand to the low back, and fingers were placed between the mat and the participants' anterior superior iliac spine (on the side of the thigh being raised). Exercises 3 and 4 involved various hip abduction and hip external rotation positions; hip external rotation range varied, therefore each participant's maximum range of motion was used. While lying on the contralateral side to the exercising leg, participants abducted the hip against a rigid adjustable bar frame (MotionBlock ${ }^{\mathrm{TM}}$ ) that was placed at the appropriate distance above the participant to ensure a $30^{\circ}$ abduction angle position. For exercise 5 , lying prone on the MotionBlock ${ }^{\mathrm{TM}}$, the participant attempted knee flexion at a fixed angle of $90^{\circ}$ against the adjustable bar frame (MotionBlock ${ }^{\mathrm{TM}}$ ). Lateral thigh movement during the contraction was limited by positioning the body beside the back wall of the MotionBlock ${ }^{\mathrm{TM}}$ table. The participant was instructed to keep the exercising leg "lightly touching the wall".

\section{Statistical Analyses}

An a priori power analysis calculation showed that a participant pool of 22 was required in order to achieve a power level of 0.8. The effect size of 0.6 was based on data and power analyses done in similar studies on lower extremity kinematic changes seen with training (Earl-Boehm et al., 2011; Hewett et al., 2005; Joseph et al., 2008; Snyder, Earl, O'Connor, \& Ebersole, 2009). Given that the convenience sample of 14 participants was anticipated to not likely result in statistical significance (i.e., because the study was underpowered for group-level analyses), the results were assessed on both a group and individual basis. Two-way mixed ANOVAs with one between-participant factor (Group = TG vs. CG) and one within-participant factor (Time = pre- vs. post-training) were performed to test for mean differences in the dependent variables (i.e., peak knee valgus angles and muscle strength measures). ANOVAs were performed using SAS system software for Windows (Version 9.3.1, SAS Institute Inc., Cary, NC), with the alpha level set at 0.05 .

Single-participant analyses were conducted using a model statistic procedure (Bates, Dufek, \& Davis, 1992). The basic approach was to compare differences between dependent variables in the pre- and post-testing conditions to that of a probabilistic critical difference (test statistic) on a participant-by-participant basis (Bates, James, \& Dufek, 2004). If the empirically observed mean difference $\left(\mid\right.$ mean $_{\text {pre }}-$ mean $_{\text {post }} \mid$ ) was greater than the test statistic (critical value $x$ $\left[\mathrm{sd}_{\text {pre }}^{2}+\mathrm{sd}_{\text {post }}^{2} / 2\right]^{1 / 2}$, where $\mathrm{sd}=$ standard deviation) for a given participant, then the difference was deemed statistically significant for that participant. Critical values based on the number of trials collected and desired alpha levels $(0.01$, 0.05 , or 0.10 ) were garnered from a table generated by Bates et al. (1992). Single-participant analyses were performed using Microsoft Excel software.

\section{RESULTS}

There were significant group $\times$ time interaction effects for hip extension (left $\mathrm{P}=0.0483$, right $\mathrm{P}=0.0085$ ), hip abduc- tion (left $\mathrm{P}=0.0036$, right $\mathrm{P}=0.0255$ ), and knee flexion (left $\mathrm{P}=0.0026$, right $\mathrm{P}=0.0010$ ) strength from pre- to post-test in the TG. On average (bilaterally), hip extension, hip abduction, and knee flexion force increased by $20.5 \%, 27.5 \%$ and $23.5 \%$, respectively (Figure 3 ). No significant changes in strength were seen in the CG. Knee extension strength did not significantly change in either group from pre- to post-test (left $\mathrm{P}=0.3018$, right $\mathrm{P}=0.6871$ ).

At the group-level, there were no significant changes in the mean peak knee valgus angle (left $\mathrm{P}=0.1038$, right $\mathrm{P}=0.375$ ) from pre- to post-test. In the individual-level analyses, five out of seven TG participants exhibited a significant decrease in peak knee valgus angle on one or both sides from pre- to post-test. Four TG participants significantly decreased their left peak knee valgus deviation (decrease of $8.3^{0}, 7.2^{0}, 2.3^{\circ}$, and $1.3^{\circ}$ ), and three TG participants significantly decreased their right knee valgus deviation (decrease of $11^{0}, 6.8^{\circ}$, and $1.9^{\circ}$ ) (Table 3 ). Of the five participants who exhibited decreases, two demonstrated a bilateral decrease in knee valgus angle (left knee and right knee valgus angles decreased by $8.3^{\circ}$ and $7.2^{\circ}$, and $11^{\circ}$ and $6.8^{\circ}$, respectively) (Figure 4). Among the CG, five, two and no participants exhibited unilateral increases (of 2.2 $2^{0}, 1.3^{0}, 2^{0}, 1^{0}$ and $2.2^{0}$ on average), decreases (of $2.3^{\circ}$ and $2.5^{\circ}$ ), and bilateral decreases in knee valgus angle.

\section{DISCUSSION}

It was hypothesized that isometric strength training of the hip extensor, abductor, and external rotator muscles would decrease the knee valgus angle during a drop vertical jump task. After a 6-week isometric strength training protocol targeting the hip extensor, abductor, and external rotator muscles, a significant improvement in strength in these muscles was seen in the training group (TG). The mean peak knee valgus angle did not change significantly in either group after training. However, significant individual improvements were seen in 5 out of 7 TG participants (two of which showed a bilateral improvement), and significant negative effects were seen in 5 out of 7 CG participants (note that two CG participants displayed a unilateral improvement in valgus movement).

The strength produced by the hip musculature (extension, hip abduction, and lying prone knee flexion - targeting the

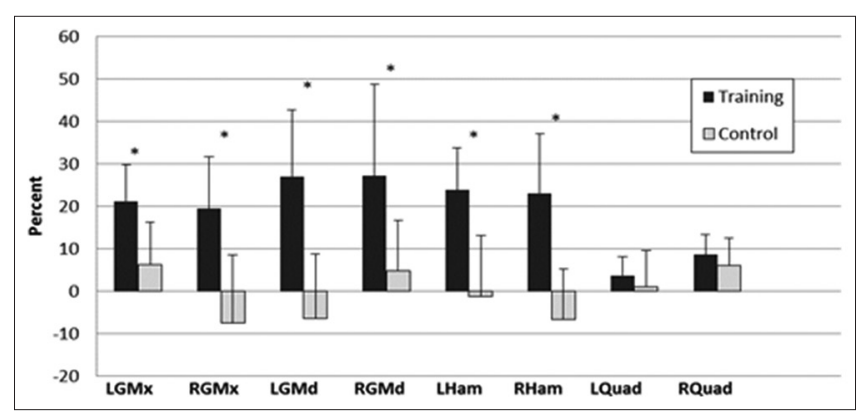

Figure 3. Percent change (error bars $=$ standard deviation) in muscle strength measured against a digital force gauge for the training group and control group between the pre- and post-test. A positive value indicates an increase in strength from the pre- to post-test 


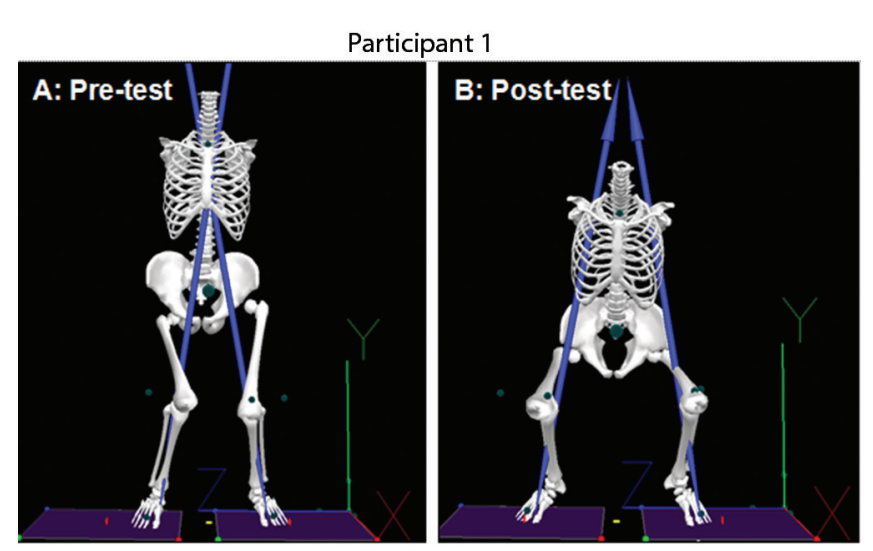

Participant 3
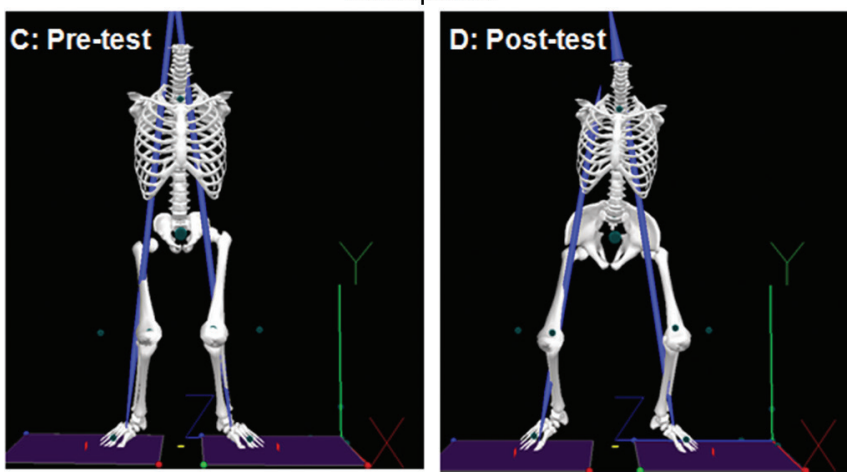

Figure 4. Frontal view at the time of peak knee valgus angle during the initial landing in pre- and post-testing for Participant 1 (pre-test $=\mathrm{A}$ and post-test $=\mathrm{B}$ ) and Participant 3 (pre-test $=\mathrm{C}$ and post-test $=\mathrm{D})$

gluteus maximus, gluteus medius, and hamstrings, respectively) improved significantly by approximately $20.5 \%$, $27.5 \%$, and $23.5 \%$. The percent strength increases seen in this and other isometric training studies were notably higher than increases reported in longer duration dynamic strength training protocols: mean increases of $7 \%$ for 12 weeks of training (Delecluse, Roelants, \& Verschueren, 2003) and 15.1\% for 10 weeks of training (Painter et al., 2012). The results of the current study support the use of isometric training over a 6-week duration as a means by which significant strength gains can be achieved at the trained joint angle. A shorter time to achieve larger strength gains would be beneficial among those seeking to improve strength as fast as possible, such as athletes, and patients undergoing rehabilitation. The isolation of the target muscle groups seems to be an ideal solution to increase the strength without strengthening surrounding non-target muscle groups (i.e., strengthening the gluteal muscles without strengthening the quadriceps), if desired to remedy muscle strength imbalances.

Group-wide, there was no significant change in peak knee valgus angle after training. The training protocol in this study proved to be adequate to generate significant strength gains of the target musculature, thereby allowing for possible connections to be made between changes in strength and changes in kinematics. However, the overall lack of significant group changes in knee valgus angle suggests that isometrically stronger muscles around the hip joint do not necessarily translate into significant changes in dynamic knee kinematics of a DVJ task for the population studied. These findings could also suggest that the changes observed after isometric training may vary greatly based on each individual, and may require that movement (re)training using combined approaches is needed to more consistently facilitate positive transfer of strength gains (Donnell-Link et al., 2015).

When considered on an individual level, five training group participants exhibited significantly lower peak knee valgus angles for one or both knees following the intervention. Lower knee valgus angles are associated with a decrease in the strain on the ACL and a lower injury risk (Hewett et al., 2005). No control group participants exhibited a bilateral decrease in knee valgus angle, although two demonstrated a unilateral decrease in the right knee. This suggests that isometric strengthening of the hip extensors, external rotators, and abductors could potentially improve the frontal plane kinematics during a drop vertical jump task in certain individuals. However, due to the low number of participants, the effectiveness of the isometric training protocol on frontal plane lower extremity kinematics is inconclusive in this study. The lack of a significant group-wide change in knee valgus angles, or even a consistent trend amongst all training group participants, suggests that strengthening the hip musculature may affect each athlete differently. An exercise program's effectiveness could vary from person to person as each individual may differ in unique physiological and psychological factors that affect performance and response to exercise (Cowley, Ford, Myer, Kernozek, \& Hewett, 2006; Eynon et al., 2011; Miller, MacDougall, \& Sale, 1993). In addition, gains in isometric strength of specific muscle groups (through position isolation) associated with poor landing kinematics may not generally transfer to dynamic movement patterns. Increasing the isometric strength of the hip muscles could perhaps serve as an initial step in a program which utilizes the benefits of dynamic or neuromuscular training in conjunction with isometric training, since a combined approach has been previously shown to be effective (Donnell-Link et al., 2015; Fontenay et al., 2013). Future studies should assess the efficacy of utilizing a combined intervention with isometric and dynamic resistance training.

One limitation was the low number of participants (14 total), with only seven participants who completed the training program. Based on a priori statistical power calculations, it was acknowledged that the study would be under-powered at the outset. As a consequence, it was decided to collect 15 DVJ trials during pre- and post-testing to combine individual- and group-level statistical analyses, which can be considered a strength of the study. It is possible that the lack of significant findings was due to small numbers, and/or attributable to the variability with which the participants executed the DVJ task. There were some trends seen for a number of individual participants in both the TG and CG regarding VA, but no significant group effects were seen. Similar studies assessing the mechanics of a DVJ task after a training protocol had participant pools of 30 (Chappell et al., 2008), 19 (Earl-Boehm et al., 2011), 50 (Cochrane et al., 2010) and 74 (Herman et al., 2008) individuals. Despite our best efforts to recruit, 68 out of 109 athletes did not meet the inclusion criteria. Of the 41 athletes remaining, only 7 were able to commit to the time-intensive nature of the intervention (i.e., 5 
Table 3. Mean (standard deviation) 3D peak knee valgus angles for the training (TG) and control (CG) group participants in pre- to post-testing

\begin{tabular}{|c|c|c|c|c|c|c|c|c|c|c|c|}
\hline \multirow{4}{*}{$\begin{array}{l}\text { Group } \\
\text { TG }\end{array}$} & \multirow{4}{*}{$\begin{array}{c}\text { ID } \\
\\
1\end{array}$} & \multicolumn{10}{|c|}{ Mean (SD) Knee Valgus Angle (deg) } \\
\hline & & \multicolumn{5}{|c|}{ Left side } & \multicolumn{5}{|c|}{ Right side } \\
\hline & & \multicolumn{2}{|c|}{ Pre } & \multicolumn{2}{|c|}{ Post } & \multirow{2}{*}{$\frac{\text { P-value }}{<0.001}$} & \multicolumn{2}{|c|}{ Pre } & \multicolumn{2}{|c|}{ Post } & \multirow{2}{*}{$\frac{\text { P-value }}{<0.001}$} \\
\hline & & 4.3 & $(2.9)$ & -4.0 & $(2.3)^{*}$ & & -13.2 & $(1.9)$ & -2.2 & $(4.9)^{*}$ & \\
\hline & 3 & 2.7 & $(1.7)$ & -4.5 & $(6.1)^{*}$ & $<0.001$ & -3.8 & $(2.1)$ & 3.0 & $(6.7)^{*}$ & $<0.001$ \\
\hline & 4 & 7.2 & $(1.7)$ & 7.2 & $(1.4)$ & 0.9893 & -7.6 & $(2.0)$ & -5.7 & $(1.8)^{*}$ & 0.0114 \\
\hline & 5 & -1.9 & (2.6) & -4.2 & $(2.2)^{*}$ & 0.0125 & -4.9 & $(1.6)$ & -4.3 & $(2.6)$ & 0.4241 \\
\hline & 6 & 0.6 & $(2.1)$ & 0.7 & $(1.6)$ & 0.8902 & -0.6 & (3.3) & -1.4 & $(2.6)$ & 0.5018 \\
\hline & 8 & 2.1 & (2.1) & 7.1 & $(1.7)^{*}$ & $<0.001$ & 0.2 & $(2.5)$ & -2.8 & $(1.9)^{*}$ & $<0.001$ \\
\hline & 14 & 1.8 & (1.6) & 0.5 & $(1.7)^{*}$ & 0.0514 & -2.3 & (1.8) & -3.8 & $(1.5)^{*}$ & 0.0173 \\
\hline \multirow[t]{7}{*}{$\mathrm{CG}$} & 2 & 0.1 & (1.7) & 2.3 & $(2.6)^{*}$ & 0.0320 & -2.6 & $(2.0)$ & -3.6 & $(1.5)$ & 0.1120 \\
\hline & 7 & 0.4 & (1.4) & 1.7 & $(2.0)^{*}$ & 0.0364 & 2.2 & (1.9) & 1.6 & $(2.5)$ & 0.4492 \\
\hline & 9 & 0.1 & (2.0) & 0.9 & $(2.3)$ & 0.3407 & -0.4 & (1.1) & 0.2 & $(1.3)$ & 0.1562 \\
\hline & 10 & 2.0 & (1.6) & 4.0 & $(2.1)^{*}$ & 0.0061 & -3.8 & (1.1) & -3.6 & $(2.2)$ & 0.8243 \\
\hline & 11 & -0.4 & (1.4) & -1.2 & $(1.4)$ & 0.1579 & -0.2 & (1.3) & 2.5 & $(1.4)^{*}$ & $<0.001$ \\
\hline & 12 & 5.1 & (2.6) & 6.1 & $(2.2)$ & 0.2795 & -2.4 & $(2.2)$ & -4.6 & $(2.3)^{*}$ & 0.0122 \\
\hline & 13 & 4.6 & (1.7) & 5.6 & $(1.2)^{*}$ & 0.0585 & -2.4 & $(1.0)$ & 0.1 & $(1.6)^{*}$ & $<0.001$ \\
\hline
\end{tabular}

Positive left side and negative right side value = valgus; negative left side and positive right side value $=$ varus

sessions/week for 6 weeks) due to their volleyball training and competition schedule. It was due to the smaller-than-desired sample size that despite collecting force plate data, we a posteriori elected against conducting inverse dynamics analyses; analyzing joint kinetics would have necessitated the application of additional correction factors, which would have further limited our ability to detect changes. Future studies should be designed with sufficient sample sizes to test the effect of isometric strength training on lower extremity joint kinetic and/or muscle activation patterns (using electromyography) during the DVJ.

Another limitation was the inability to strictly control the athletes' activities outside of the isometric training protocol, again due to their competition and training schedule. However, athletes were asked to continue their regular level of volleyball play, and were discouraged from making any changes in their current fitness training. There was a high level of adherence to these requests, established by verbal confirmation.

The reliability of the anatomical landmarking needed for the motion capture markers utilized during the pre- and post-test data collections was controlled by having the same researcher perform the task in all cases. However, the variability in marker placement was not evaluated independently in this study. The overall impact that differences in marker placement has on knee valgus angle calculations pre- and post-test should be considered in future studies, especially given that a number of differences were noted in the pre- and post-test knee valgus angles in the $\mathrm{CG}$.

The participants played volleyball at a highly competitive level. Consequently, their movement patterns (dropjump skill) may have been more engrained than a younger, less trained cohort, and could therefore be more resistant to change after a 6-week isometric strength training program.
This was not seen as a major limitation to the current study in that the focus was on changing knee valgus angle in competitive players. The changes that were noted for a few individuals are encouraging, and will hopefully translate into reduced injury risk for them. This is viewed as a positive outcome of this work, and a starting point for future studies in this area.

\section{CONCLUSIONS}

Although hip extensor, abductor, and external rotator muscle weaknesses are associated with high-risk DVJ mechanics (i.e., uncontrolled frontal plane knee motion), isometric strengthening of these muscle groups did not directly transfer to improved DVJ mechanics based on the group-level analyses conducted in this study. However, improvements in DVJ mechanics were detected in individual participants, which warrant further investigation of the effectiveness of isometric training at the individual level. Moreover, using single-participant experimental designs, future studies should assess combinations of isometric and neuromuscular training to potentially enhance the effectiveness of training to alter DVJ mechanics, and therefore reduce ACL injury risk.

\section{REFERENCES}

Anwer, S., \& Alghadir, A. (2014). Effect of isometric quadriceps exercise on muscle strength, pain, and function in patients with knee osteoarthritis: a randomized controlled study. Journal of Physical Therapy Science, 26(5), 745-748.doi:10.1589/ipts.26.745

Bates, B.T., Dufek, J.S., \& Davis, H.P. (1992). The effect of trial size on statistical power. Medicine and Science in Sports and Exercise, 24(9), 1059-1068. doi: 10.1249/00005768-199209000-00017 
Bates, B.T., James, C.R., \& Dufek, J.S. (2004). Single-subject analysis. In N. Stergiou (Ed.), Innovative Analyses of Human Movement (pp. 3-28). Champaign, IL: Human Kinetics. 3-28.

Bessier, T.F., Lloyd, D.G., Cochrane, J.L., Ackland, T.R. (2001). External loading of the knee joint during running and cutting maneuvers. Medicine and Science in Sport and Exercise, 33, 1168-1175. doi:10.1097/00005768200107000-00014

Boden, B.P., Dean, G.S., Feagin, J.A. Jr., Garrett, W.E. Jr. (2000). Mechanisms of anterior cruciate ligament injury. Orthopedics, 23, 573-578. doi: 10.3928/0147-744720000601-15

Butler, D. L., Noyes, F. R., \& Grood, E. S. (1980). Ligamentous restraints to anterior-posterior drawer in the human knee. The Journal of Bone and Joint Surgery, 62(2), 259-270. Retrieved from http://jbjs.org.myaccess.library.utoronto.ca/content/jbjsam/62/2/259.full.pdf

Cammarata, M. L. \& Dhaher, Y. Y. (2010). Evidence of gender-specific motor templates to resist valgus loading at the knee. Muscle \& Nerve, 41(5), 614-623. doi:10.1002/ mus. 21509

Chappell, J.D. \& Limpisvasti, O. (2008). Effect of a neuromuscular training program on the kinetics and kinematics of jumping tasks. The American Journal of Sports Medicine, 36(6), 1081-1086. doi:10.1177/0363546508314425

Cochrane, J. L., Lloyd, D. G., Besier, T. F., Elliott, B. C.m Doyle, T. L. A., \& Ackland, T. R. (2010). Training affects knee kinematics and kinetics in cutting maneuvers in sport. Medicine and Science in Sport and Exercise, 42(8), 1435-1544. doi:10.1249/MSS.0b013e3181d$03 \mathrm{ba} 0$

Cooke, T. D. V., Sled, E. A., \& Scudamore, R. A. (2007). Frontal plane knee alignment: A call for standardized measurement. Journal of Rheumatology, 34(9), 17961801. Retrieved from https://jrheum.com/subscribers/07/09/1796.html

Cowley, H. R., Ford, K. R., Myer, G. D., Kernozek, T. W., \& Hewett, T. E. (2006). Difference in neuromuscular strategies between landing and cutting tasks in female basketball and soccer athletes. Journal of Athletic Training, 41(1), 67-73. Retrieved from https://www.ncbi.nlm.nih. gov/pmc/articles/PMC1421490

de Loes, M., Dahlstedt, L.J., \& Thomee, R. (2000). A 7-year study on risks and costs of knee injuries in male and female youth participants in 12 sports. Scandinavian Journal of Medical Science and Sports, 10(2), 90-97. doi: 10.1034/j.1600-0838.2000.010002090.x

Delecluse, C., Roelants, M., \& Verschueren, S. (2003). Strength increase after whole-body vibration compared with resistance training. Medicine and Science in Sports and Exercise, 6, 1033-1041. doi:10.1249/01. MSS.0000069752.96438.B0

Donnell-Link, L. A., Klara, K., Collins, J. E., Yang, H. Y., Goczalk, M. G., Katz, J. N., \& Losina, E. (2015). Effectiveness of knee injury and anterior cruciate ligament tear prevention programs: A meta-analysis. PLoS One, 10(12), e0144063. doi:10.1371/journal.pone.0144063
Duchateau, J. \& Hainaut, K. (1984). Isometric or dynamic training: differential effects on mechanical properties of a human muscle. Journal of Applied Physiology, 56(2), 296-301. Retrieved from http://jap.physiology.org/content $/ 56 / 2 / 296$

Earl-Boehm, J. E. \& Hoch, A. Z. (2011). A proximal strengthening program improves pain, function, and biomechanics in women with patellofemoral pain syndrome. The American Journal of Sports Medicine, 39(1), 154-163. doi:10.1177/0363546510379967

Ellison, A. E. \& Berg, E. E. (1985). Embryology, anatomy, and function of the anterior cruciate ligament. Orthopedic Clinics of North America, 16, 3-14. Retrieved from http://search.proquest.com.myaccess.library.utoronto. $\mathrm{ca} /$ docview $/ 75955310$ ?accountid $=14771$

Eynon, N., Ruiz, J. R., Oliveira, J., Duarte, J. A., Birk, R., \& Lucia, A. (2011). Genes and elite athletes: a roadmap for future research. The Journal of Physiology, 589, 30633070. doi:10.1113/jphysiol.2011.207035

Ferber, R., Kendall, K. D., \& Farr, L. (2011). Changes in knee biomechanics after a hip-abductor strengthening protocol for runners with patellofemoral pain syndrome. Journal of Athletic Training, 46(2), 142-149. doi:10.4085/1062-6050-46.2.142

Fernandez, W. G., Yard, E. E., \& Comstock, R. D. (2007). Epidemiology of lower extremity injuries among U.S. high school athletes. Academic Emergency Medicine: Official Journal of the Society for Academic Emergency Medicine, 14(7), 641-645. doi:10.1197/j.aem.2007.03.1354

Folland, J. P., Hawker, K., Leach, B., Little, T., \& Jones, D. A. (2005). Strength training: Isometric training at a range of joint angles versus dynamic training. Journal of Sports Sciences, 23(8), 817-824. doi: $10.1080 / 02640410400021783$

Fontenay, B.P.D., Lebon, F., Champely, S., Argaud, S., Blache, Y., Collet, C., Monteil, K. (2013). ACL injury risk factors decrease $\&$ jumping performance improvement in female basketball players: a prospective study. International Journal of Kinesiology \& Sports Science, 1(2), 10-18. doi:10.7575/aiac.ijkss.v.1n.2p.10

Ford, K. R., Myer, G. D., \& Hewett, T. E. (2003). Valgus knee motion during landing in high school female and male basketball players. Medicine \& Science in Sport \& Exercise, 35(10), 1745-1750. doi:10.1249/01. MSS.0000089346.85744.D9

Herman, D. C., Weinhold, P. S., Guskiewicz, K. M., Garrett, W. E., Yu, B., \& Padua, D. A. (2008). The effects of strength training on the lower extremity biomechanics of female recreational athletes during a stop-jump task. The American Journal of Sports Medicine, 36(4), 733-740. doi:10.1177/0363546507311602

Hewett, T.E., Myer, G.D., Ford, K.R., Heidt, R.S., Colosimo, A.L., McLean, S.G., van den Bogert, A.J., Paterno, M.V., \& Succop, P. (2005). Biomechanical measures of neuromuscular control and valgus loading of the knee predict anterior cruciate ligament injury risk in female athletes. The American Journal of Sports Medicine, 33(4), 492-501. doi:10.1177/0363546504269591 
Howard, J. (2011). Structure, sex, and strength and knee and kinematics during landing. Journal of Athletic Training, 46(4), 376-385. Retrieved from http://www.natajournals.org/doi/full/10.4085/1062-6050-46.4.376? code $=$ nata-site

Jacobs, C. A., Uhl, T. L., Mattacola, C. G., Shapiro, R., \& Rayens, W. S. (2007). Hip abductor function of lower extremity landing kinematics: Sex differences. Journal of Athletic Training, 41(1), 76-83. Retrieved from https:// www.ncbi.nlm.nih.gov/pmc/articles/PMC1896084

Joseph, M. F., Rahl, M., Sheehan, J., MacDougall, B., Horn, E., Denegar, C. R., Trojian, T. H., Anderson, J. M., \& Kraemer, W. J. (2011). Timing of lower extremity frontal plane motion differs between female and male athletes during a landing task. The American Journal of Sports Medicine, 39(7), 1517-1521. doi:10.1177/0363546510397175

Joseph, M., Tiberio, D., Baird, J. L., Trojian, T. H., Anderson, J. M., Kraemer, W. J., \& Maresh, C. M. (2008). Knee valgus during drop jumps in national collegiate athletic association division I female athletes: The effect of a medial post. The American Journal of Sports Medicine, 36(2), 285-289. doi:10.1177/0363546507308362

Khayambashi, K., Mohammadkhani, Z., Ghaznavi, K. Lyle, M. A., \& Powers, C. M. (2012). The effects of isolated hip abductor and external rotator muscle strengthening on pain, health status, and hip strength in females with patellofemoral pain: A randomized controlled trial. The Journal of Orthopaedic and Sports Physical Therapy, 42(1), 22-29. doi:10.2519/jospt.2012.3704

Khayambashi, K., Ghoddosi, N., Straub, R.K., Powers, C.M. (2015). Hip muscle strength predicts noncontact anterior cruciate ligament injury in male and female athletes: A prospective study. The American Journal of Sports Medicine, 44(2), 355-361. doi:10.1177/0363546515616237

Khuu, S., Musalem, L. L., \& Beach, T. A. (2015). Verbal instructions acutely affect drop vertical jump biomechanics-implications for athletic performance and injury risk assessments. Journal of Strength and Conditioning Research, 29(10), 2816-26. doi:10.1519/ JSC.0000000000000938

Kristianslund, E., Krosshaug, T., \& van den Bogert, A. J. (2012). Effect of low pass filtering on joint moments from inverse dynamics: Implications for injury prevention. Journal of Biomechanics, 45, 666-671. doi:10.1016/j. jbiomech.2011.12.011
Levine, J. W., Kiapour, A. M., Quatman, C. E., Wordeman, S. C., Goel, V. K., Hewett, T. E. \& Demetropoulos C. K. (2013). Clinically relevant injury patterns after an anterior cruciate ligament injury provide insight into injury mechanisms. The American Journal of Sports Medicine, 41(2), 385-395. doi:10.1177/0363546512465167

Miller, A. E. J., MacDougall, M. A., \& Sale, D. G. (1993). Gender differences in strength and muscle fiber characteristics. European Journal of Applied Physiology, 66, 254-262. doi:10.1007/BF00235103

Myer, G. D., Ford, K. R., Palumbo, J. P., \& Hewett, T. E. (2005). Neuromuscular training improves performance and lower-extremity biomechanics in female athletes. Journal of Strength \& Conditioning Research, 19(1), 51-60. doi:10.1519/13643.1

Myer, G., Ford, K. R., McLean, S. G., \& Hewett, T. E. (2006). The effects of plyometric versus dynamic stabilization and balance training on lower extremity biomechanics. The American Journal of Sports Medicine, 34(3), 445-455. doi: 10.1177/0363546505281241

Nadler, S. F., Malanga, G. A., Solomona, J. L., Feinberg, J. H., Foyea, P. M., \& Park, Y. I. (2002). The relationship between lower extremity injury and the hip abductor to extensor strength ratio in collegiate athletes. Journal of Back and Musculoskeletal Rehabilitation, 16, 153-158. doi:10.3233/BMR-2002-16406

Painter, K. B., Haff, G. G., Ramsey, M. W., McBride, J., Triplett, T., Sands, W. A., Lamont, H. S., Stone, M. E., \& Stone, M. H. (2012). Strength gains: Block versus daily undulating periodization weight training among track and field athletes. International Journal of Sports Physiology and Performance, 7, 161-169. doi:10.1123/ ijspp.7.2.161

Powers, C. M. (2010). The influence of abnormal hip mechanics on knee injury: a biomechanical perspective. Journal of Orthopaedic \& Sports Physical Therapy, 40(2), 42-51. doi:10.2519/jospt.2010.3337

Snyder, K. R., Earl, J. E., O’Connor, K. M., \& Ebersole, K. T. (2009). Resistance training is accompanied by increases in hip strength and changes in lower extremity biomechanics during running. Clinical Biomechanics, 24(1), 26-34. doi:10.1016/j.clinbiomech.2008.09.009

Tsao, H., Galea, M., \& Hodges, P. (2010). Driving plasticity in the motor cortex in recurrent low back pain. European Journal of Pain, 14, 832-839. doi:10.1016/j. ejpain.2010.01.001 